\title{
认知无线网络中基于共识理论的分布式聚类合作 频谱感知研究
}

\author{
吴启晖"，丁国如"，王金龙，李小强，黄育侦 \\ 解放军理工大学通信工程学院, 南京 210007 \\ *联系人, E-mail: wqhqhw@163.com; guoru_ding@yeah.net \\ 2011-11-01 收稿, 2012-01-11 接受 \\ 国家重点基础研究发展计划(2009CB320400)、国家自然科学基金(60932002 和 61172062)和江苏省自然科学基金(SBK201122196)资助
}

\begin{abstract}
摘要 大量的已有研究工作表明：多用户合作频谱感知可以明显提升检测性能. 然而, 当网 络中用户数目较多, 并且用户所分布的空间范围较大时, 所有用户均参与合作将会带来巨大 的感知开销(例如感知时间和能量消耗). 基于无监督学习技术和共识理论的最新进展, 在本 文中我们提出了一种全分布式的合作频谱感知方案. 在所提方案中, 仅通过一跳可达的邻居 之间的信息交互，具有潜在最佳检测性能的用户会自组织地聚到一起，这些用户进而利用平 均共识协议来进行合作频谱感知, 然后将感知结果广播至全网用户。为了进行性能比较, 进 一步给出了最优软合并的一种分布式实现方案. 数值结果表明: 所提方案获得了与最优软合 并方案相近的检测性能，并明显优于已有等增益合并方案和基于位置信息的方案．同时，相 比于最优软合并方案, 所提方案可以大幅度降低感知开销, 并且不需要关于用户本地信噪比 的先验信息.
\end{abstract}

\section{关键词}

认知无线网络

频谱感知

分布式聚类

无监督学习

共识理论
认知无线网络 (cognitive radio networks, CRNs) 中, 次级用户 (secondary users, SUs)机会地利用主用 户(primary users, PUs)留下的频谱空洞 ${ }^{[1]}$ 进行通信来 提升无线频谱利用率. 为了找到时域或/和空域频谱 洞，面临的关键挑战之一是实现可靠且有效的频谱 感知. 相比于单节点频谱感知方法 ${ }^{[2]}$, 多个次级用户 合作来进行频谱感知可以利用多用户空间分集，从

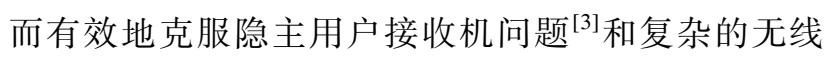
信道环境(如阴影衰落和多径衰落) ${ }^{[4]}$. 文献[5]和[6]对 合作频谱感知(cooperative spectrum sensing, CSS)的 最新进展进行了系统的综述.

从是否存在融合中心的角度来看, 目前关于 CSS 的研究主要分为两类: 中心式 CSS 和分布式 CSS. 已有的大量研究工作集中在中心式 CSS 的方案 设计上, 包括硬判决合并方案 ${ }^{[8 \sim 10]}$, 软信息合并方 案 ${ }^{[11,12]}$ 和量化信息合并方案 ${ }^{[13]}$ 等. 为进一步降低感
知开销(如感知时间和能量消耗), 提升网络可扩展性 和稳健性，分布式(或无融合中心)CSS 成为近期研究 的热点. 在文献[14]中，作者把分布式 CSS 建模为一 个进化博弯问题, 每个 SU 通过与其他 SU 之间的交 互策略来学习自身最佳的策略值(合作或不合作)。注 意到文献[14]中考虑的仅是一跳网络, 即网络中的所 有用户都互为邻居. 在文献[15]中, 分布式 CSS 问题 通过联盟形成博弯来解决, 其中每个 SU 通过权衡加 人联盟前后收益与代价来决定自己的策略(加人联盟 或不加人). 注意到文献[15]中的方案中存在联盟头, 因此，其方案不是全分布式的. 基于平均共识理论, 文献[16]的作者提出了一种全分布式的 CSS 方案, 其 中每个 SU 迭代地与一跳可达邻居进行感知信息交互, 直到全网对感知信息形成平均共识. 注意到文献 [16] 本质上属于传统的基于软信息等增益合并(equal gain combination, EGC)中心式 $\mathrm{CSS}^{[12]}$ 的分布式实现.

英文版见: Wu Q H, Ding G R, Wang J L, et al. Consensus-based decentralized clustering for cooperative spectrum sensing in cognitive radio networks. Chin Sci Bull, 2012, 57, doi: 10.1007/s11434-012-5074-6 
综上，尽管近年来 CSS 问题得到了广泛而深人 的研究, 目前仍然有许多问题亟待解决. 首先, 当所 考虑的 CRNs 规模较大时, 即网络中 SUs 的数目和它 们所分布的地理区域较大时, 如果所有 SUs 均参与 合作，那么合作将会带来巨大的网络开销. 其次，考 虑路径损耗和阴影衰落等因素, 处于不同位置的 SUs 的检测可靠性也会存在显著的区别. 尽管目前存在 相关的研究来考虑类似的问题, 如节点选择 ${ }^{[18]}$ 、基于 分簇的 $\mathrm{CSS}^{[19]}$ 等, 但至今还没有全分布式(即无任何 中心协调器)的方案. 因此, 基于无监督学习理论和 共识理论的最新进展，本文致力于设计一种基于分 布式聚类的全分布式 CSS 方案.

无监督学习理论 ${ }^{[20]}$ 是模式识别和人工智能领域 的重要理论之一, 其核心思想是“不需要导师的自 学”, 故与分布式网络数据融合计算有着内在的共性. 而共识理论 ${ }^{[17]}$ 最初来源于生物群体智能现象, 例如 雁群飞行, 蜂群采蜜等, 近年来在分布式控制与决 策、多智能体合作和传感器网络分布式参数估计等领 域得到广泛的研究.

相比于已有研究工作, 本文所提方案具有如下 3 个新的特征:

(1) 网络操作是全分布式的. 在所提方案中, 不 需要任何的中心协调器 (如基站, 接人点, 簇头等), 所有的信息交互仅在一跳可达的邻居之间进行. 因 此, 所提方案具备抗单点失败稳健性好、网络可扩展 性好以及网络开销小等优点.

（2）所提算法的复杂度很低. 一方面, 所提算法 中每个 SU 不需要进行其自身接收信噪比的估计, 也 不需要主用户位置的先验信息; 另一方面, 算法不需 要额外的时间开销来获取聚类所需的观测量, 因为 该观测量可以通过离线的方式学习得到。

(3) 所提算法在获取较高检测可靠性的同时, 大 大降低了网络开销. 仿真显示, 本文所提算法可以获 得与最优软合并方案(optiaml soft combination, OSC) 相近的检测性能, 且所提方案仅需要部分 SU 参与合 作, 不需要进行瞬时信噪比的估计.

\section{1 系统模型和问题陈述}

\section{1 网络模型和假设}

我们考虑的网络由 1 个 PU 和 $N$ 个 SUs 组成. 不 失一般性, 我们假设 PU 位于 $L \times L$ 正方形区域的中心,
$N$ 个 SUs 均匀地分布在该区域内. 注意到, 下文所提 方案可以进一步拓展到网络中存在多个 PU 的场景. 我们将网络建模为一个无向图 $G=(V, E)$, 其中 $V$ 表 示顶点集，每个顶点对应一个 $\mathrm{SU}, E$ 表示顶点之间 的边，每条边反映 SU 之间的连通关系. SUs 之间的一 跳连通关系通过邻接矩阵 $\boldsymbol{A} \in R^{N \times N}$ 来刻画. 如果 $\mathrm{SU} i$ 和 $\mathrm{SU} j$ 一跳互通, 则 $\boldsymbol{A}_{i j}=\boldsymbol{A}_{j i}=1$; 否则 $\boldsymbol{A}_{i j}=\boldsymbol{A}_{j i}=0$. 我们假设 $\mathrm{SU} i$ 仅可以与一跳可达的邻居进行直接通 信, 其邻居集为 $N_{i}:=\left\{j: j \in V, \boldsymbol{A}_{i j}=1\right\}$, 其邻居的个 数称作 SU $i$ 的度, 记为 $D_{i}:=\left|N_{i}\right|$. 我们进一步假设每 个 SU 不需要 PU 和其他 SUs 的先验位置信息, 仅基 于感知到的 PU 的功率强度, $N$ 个 SUs 之间以一种自 组织的方式进行合作频谱感知来发现频谱空洞.

\section{2 信道模型和能量检测模型}

本文中, 从 PU 到 $\mathrm{SU} i$ 的瞬时信道功率增益 $g(i)$ 由 路径损耗 $p(i)$, 阴影衰落 $s(i)$ 和多径瑞利衰落 $f(i)$ 组成 ${ }^{[21]}$

$$
g(i)=p(i) \cdot s(i) \cdot f(i),
$$

其中路径损耗分量为 $p(i)=d_{S P}(i)^{-n}, d_{S P}(i)$ 表示从 $\mathrm{PU}$ 到 $\mathrm{SU} i$ 的距离, $n$ 表示路径损耗因子. 阴影衰落分量 (以 $\mathrm{dB}$ 单位, $10 \log _{10} s(i)$ ) 服从一个零均值、标准差为 $\sigma$ 的正态分布. 多径瑞利分量 $f(i)$ 服从一个均值为 $\mu$ 的指数分布. 为了简化分析, 本文假设不同信道之间 的阴影衰落和多径衰落相互独立.

在此信道模型下, 每个 $\mathrm{SU}$ 进行本地能量检测 ${ }^{[2]}$ 来确定是否存在频谱机会. 令 $x(n)$ 表示 PU 发射的信 号, $w(i, n)$ 表示 $\mathrm{SU} i$ 本地的噪声. $\mathrm{SU} i$ 本地采集到的样 值点可以表示为 $y(i, n)=h(i) x(n)+w(i, n)$. 其中 $h(i)=$ $\sqrt{g(i)}$ 瞬时的信道幅度增益. 注意到, 如果 PU 信号 不存在, 则 $x(n)$ 将恒为 0 . 故 $\mathrm{SU} i$ 采集到的信号总能 量可以表示为 $e(i)=\sum_{n=1}^{N_{s}}|y(i, n)|^{2}$.

根据中心极限定理, 如果采样点数 $N_{s}$ 足够大, 感 知到的能量将近似服从正态分布, 其均值和方差为

$$
\begin{gathered}
E[e(i)]=N_{s}[1+\gamma(i)] \delta_{i}^{2}, \\
\operatorname{Var}[e(i)]=2 N_{s}[1+2 \gamma(i)] \delta_{i}^{4},
\end{gathered}
$$

其中, $\delta_{i}^{2}$ 噪声方差, $\gamma(i)=1 / N_{s} \sum_{n=1}^{N_{s}}|x(n) h(i)|^{2} / \delta_{i}^{2}$ 为平均接受信噪比.

\section{3 问题陈述}

本文考虑一个大规模认知无线网络里的分布式 
CSS 的问题. 该网络中 SU 数目 $N$ 和 SUs 分布的地理 空间均较大. 在这样的场景下, 如果所有 SUs 都参与 合作, 将会带来巨大的感知时间和能量开销. 并且, 由于空域上的广泛分布和无线信道的复杂性，不同 的 SUs 之间的检测可靠性差别会很明显. 直观上, 距 离 PU 较近的 SUs(图 1 中虚线圈内的节点)具有较高 的可靠性. 但如果进一步考虑阴影衰落等因素, 直观 上的结论未必是可靠的.

基于以上认识, 本文的目标是设计一个复杂度 较低的、全分布式的 CSS 方案. 那么, 我们面临的第 一个挑战是: 在无中心的情况下, 如何发现检测性能 可靠的 SUs. 在传统的中心式 CSS 的研究中, 该问题 往往被建模为最优感知节点选择的问题 ${ }^{[18]}$, 然而该 问题通常是一个 NP-难组合优化问题. 缺乏中心控制 器进一步地增加了算法设计的难度. 此外, 如何设计 一种合理的协议来实现性能较好的 SUs 自组织聚集、 分布式决策也是需要考虑的问题.

\section{2 分布式合作频谱感知}

针对以上挑战, 本文中提出了一个基于共识理 论的全分布式 CSS 方案. 如图 2 所示, 该方案由 3 个 阶段组成: 聚类阶段、感知阶段和广播阶段. 聚类阶 段实现具有最佳检测可靠性的节点的自组织聚集; 感知阶段实现类内节点之间的分布式 CSS; 广播阶 段中, 将感知结果广播给类外其他节点. 令 $T_{f}$ 表示一 个基本帧的总长度, 我们定义一个基本帧由感知时 段、广播时段和传输时段组成. 注意到聚类阶段每隔 $N_{f}=T_{c} / T_{f}$ 个基本帧激活一次, 其中 $N_{f}$ 与(由节点移动 性引起的)网络拓扑变化频率有关.

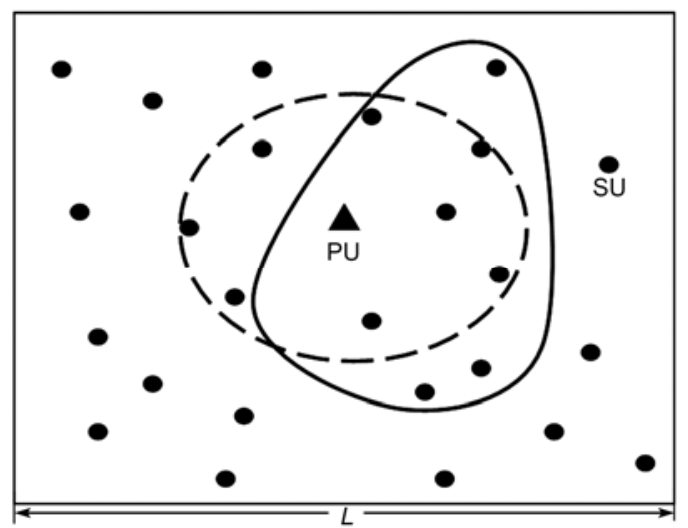

图 1 网络模型

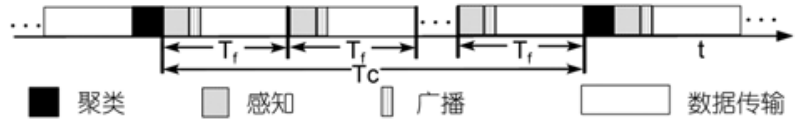

图 2 所提方案的帧结构

\section{1 阶段一: 基于共识的分布式聚类}

基于无监督学习理论和共识理论的最新进展, 我们致力于设计一种有效的算法来 “选择”感知可靠 性最好的节点. 这里的“选择”加双引号是为了强调: 本文所提方案中, 没有任何中心实体来进行全局的 控制, 实际上每个 $\mathrm{SU}$ 仅基于与一跳邻居进行信息交 互而自发地进行决策.

本文中, 我们假设网络拓扑是静态的. 因此, 每 个 $\mathrm{SU} i$ 可以通过累积连续 $M$ 个感知过程的能量值作 为聚类阶段的观测值,

$$
o(i)=\sum_{m=1}^{M} e(i, m),
$$

其中 $e(i, m)$ 表示 $\mathrm{SU} i$ 在第 $m$ 次感知过程中采集到的能 量值. 注意到聚类阶段观测值 $o(i)$ 可以离线获取, 因 此, 不需要额外的时间开销. 而且, 当 $M$ 足够大时, SUs 之间观测值的差别可以很好地反应不同 SUs 的 相对接收信号强度或本地平均接收信噪比.

基于观测值 $o(i), i \in V$, 我们提出如下的聚类方 案来实现具有最佳感知性能的节点的自组织聚集, 令 $K$ 表示类的个数(在本文中取 $K=2$ ). $a^{p}(i, k) \in[0,1]$ 表示类成员归属系数, $c(i, k)$ 表示 $\mathrm{SU} i$ 本地认识到的类 $k$ 的质心. 通过迭代地更新类成员归属系数 $\left\{a^{p}(i, k)\right\}$, 本地质心 $\{c(i, k)\}$ 和中间变量 $\{w(j, i, k)\}$, 所提方案的 目标是通过最小化偏差平方和来找到最佳类成员归 属系数, 具体建模为

$$
\begin{gathered}
\left\{a^{p^{*}}(i, k)\right\}=\underset{\substack{\left\{a^{p}(i, k)\right\},\{c(i, k)\} \\
\{w(j, i, k)\}}}{\arg \min } \frac{1}{2} \sum_{i=1}^{N} \sum_{k=1}^{K} a^{p}(i, k)\|o(i)-c(i, k)\|^{2}, \\
\text { s.t. } \quad a^{p}(i, k) \in[0,1], \forall i \in V, \forall k \in\{1, \cdots, K\} \\
\quad \sum_{k=1}^{K} a^{p}(i, k)=1, \forall i \in V \\
c(j, k)=w(j, i, k),\{w(j, i, k)\}=c(i, k) \\
\forall i \in V, \forall j \in N_{i}, \forall k \in\{1, \cdots, K\}
\end{gathered}
$$

注意到引人 $(8)$ 式的目的在于：通过算法迭代, 使得所有的 SUs 最终对各类的质心达成共识. 为了 求解上述问题, 我们首先给出其扩展拉格朗日形式, 


$$
\begin{aligned}
& L\left(\left\{a^{p}(i, k)\right\},\{c(i, k)\}\left\{\lambda_{1}(i, k)\right\},\left\{\lambda_{2}(i, k)\right\}\right) \\
= & \frac{1}{2} \sum_{i=1}^{N} \sum_{k=1}^{K} a^{p}(i, k)\|o(i)-c(i, k)\|^{2}+\sum_{i=1}^{N} \sum_{k=1}^{K} \sum_{j \in N_{i}}\left[\lambda_{1}(i, k)(c(j, k)-w(j, i, k))+\lambda_{2}(i, k)(w(j, i, k)-c(i, k))\right] \\
+ & \frac{\eta}{2} \sum_{i=1}^{N} \sum_{k=1}^{K} \sum_{j \in N_{i}}\left(\|c(j, k)-w(j, i, k)\|^{2}+\|w(j, i, k)-c(i, k)\|^{2}\right),
\end{aligned}
$$

其中, $\lambda_{1}(i, k)$ 和 $\lambda_{2}(i, k)$ 表示拉格朗日乘子, $\eta$ 是一个可调的正数.

进一步, 上述问题的对偶问题可以表示为

$$
\begin{aligned}
\min _{\left\{\lambda_{1}(i, k)\right\},\left\{\lambda_{2}(i, k)\right\}}\left\{\max _{\left\{a^{p}(i, k)\right\},\{c(i, k)\}} L\left(\left\{a^{p}(i, k)\right\},\{c(i, k)\}\left\{\left\{\lambda_{1}(i, k)\right\},\left\{\lambda_{2}(i, k)\right\}\right)\right\}\right. \\
\text { s.t. } a^{p}(i, k) \in[0,1], \forall i \in V, \forall k \in\{1, \cdots, K\} \quad \sum_{k=1}^{K} a^{p}(i, k)=1, \forall i \in V,
\end{aligned}
$$

参考文献[20], 上述对偶问题可以被渐近地分解为下 述 3 个顺序迭代的子问题.

子问题 1: 类归属系数更新

$$
\begin{aligned}
a^{p}(i, k)= & \underset{\left\{a^{p}(i, k)\right\}}{\arg \min } \frac{1}{2} \sum_{i=1}^{N} \sum_{k=1}^{K} a^{p}(i, k)\|o(i)-c(i, k)\|^{2} \\
= & \frac{\|o(i)-c(i, k)\|^{-2 /(p-1)}}{\sum_{k=1}^{K}\|o(i)-c(i, k)\|^{-2 /(p-1)}}, \\
& \forall i \in V, \forall j \in N_{i}, \forall k \in\{1, \cdots, K\}
\end{aligned}
$$

子问题 2: 节点对类的质心认识更新

$$
\begin{aligned}
c(i, k)= & \left(a^{p}(i, k)+2 \eta\left|N_{i}\right|\right)^{-1} \\
& {\left[a^{p}(i, k) o(i)-2 \lambda(i, k)+\eta \sum_{j \in N_{i}}(c(j, k)+c(i, k))\right] } \\
& \forall i \in V, \forall j \in N_{i}, \forall k \in\{1, \cdots, K\},
\end{aligned}
$$

子问题 3: 聚合拉格朗日乘子更新

$$
\begin{aligned}
& \lambda(i, k)=\lambda(i, k) \\
& \quad+\frac{\eta}{2} \sum_{j \in N_{i}}(c(j, k)-c(i, k)), \forall i \in V, \forall j \in N_{i}, \forall k \in\{1, \cdots, K\} .
\end{aligned}
$$

注意到, 参照文献[20]，(13)式中, 将拉格朗日乘子 $\lambda_{1}(i, k)$ 和 $\lambda_{2}(i, k)$ 聚合为一个乘子 $\lambda(i, k)$ 来简化计算.

通常, 经过上述算法得到的最优类归属系数 $a^{p^{*}}(i, k)$ 是一个介于 0 和 1 之间的小数. 为完成聚类 过程, 我们最后定义一个硬判决规则,

$$
a^{p^{p^{*}}}(i, k)=\left\{\begin{array}{lr}
1, & k=\arg \max _{k \in\{1, \ldots, K\}} a^{p^{*}}(i, k), \\
0, & \text { 其他. }
\end{array}\right.
$$

这样, $\mathrm{SU} i \in V^{\prime}:=\left\{i \mid a^{p^{*}}\left(i, k^{*}\right)=1, k^{*}=\arg \max _{k} c^{*}(i, k)\right\}$
将会被选中. 并且, 当上述迭代算法收玫到稳态时, 我们有 $c^{*}(i, k)=c^{*}(j, k), \forall i, j$.

\section{2 阶段二：基于共识的分布式合作频谱感知}

我们应用平均共识理论 [17]来实现所选感知节 点之间的分布式 CSS.

由于篇幅受限, 这里仅引用平均共识理论的重 要定理之一来阐明下文的算法.

定理 $1^{[17]}$ : 考虑一个由 $N$ 个用户组成的网络, 令 $x_{i}(t)$ 表示用户 $i$ 在时刻 $t$ 的共识变量, 平均共识协议 的迭代形式为:

$$
x_{i}(t+1)=x_{i}(t)+\varepsilon \sum_{j \in N_{i}}\left[x_{j}(t)-x_{i}(t)\right],
$$

其中 $0<\varepsilon<\left\{\max \left|N_{i}\right|\right\}:=1 / \Delta$ 是一迭代步长.

那么, 当网络是一个连通的无向图时, 经过有限 次迭代, 全网平均共识渐近达成, 且共识值为

$$
x^{*}=(1 / N) \sum_{i=1}^{N} x_{i}(0) .
$$

前文曾提到, 文献[16]提出了一种基于平均共识 理论的分布式 CSS 方案. 然而, 注意到在文献[16]中, 所有 SUs 均参与感知, 对于用户数目较大的网络, 这将 带来巨大的网络感知和能量开销. 并且, 一跳可达的 $\mathrm{SU}$ 之间直接交互的是采集到的能量值(即 $x_{i}(0)=e(i)$ ) 因此, 文献[16]中的算法可以看作是传统等增益合并 (EGC)方案的分布式实现 ${ }^{[12]}$. 一旦算法迭代终止, 每 个 $\mathrm{SU}$ 得到最终的共识变量值 $x^{*}$, 进行如下本地判决,

$$
\text { Decision } \boldsymbol{H}= \begin{cases}1, & x^{*}>\lambda, \\ 0, & \text { 其他, }\end{cases}
$$

其中 $\lambda$ 是一个预定义的门限值. 
我们发现，如果我们将每个用户的初始共识变 量重新设计如下:

$$
x_{i}(0)=\frac{\gamma(i)}{1+\gamma(i)} e(i), \forall i .
$$

则一旦算法迭代终止, 我们将得到中心式最优软合 并 $(\mathrm{OSC})$ 方案 ${ }^{[12]}$ 的分布式实现. 其中 $\gamma(i), \forall i \in V$ 是 $\mathrm{SU} i$ 的本地瞬时信噪比. 注意到, 通常情况下, OSC 方案的检测性能明显优于 EGC 方案, 但前者需要进 行本地信噪比的估计. 该部分的工作将作为仿真部 分性能比较的理论基础.

针对传统方案存在复杂度高和感知开销大的问 题，我们提出了如下的方案来实现分布式 CSS: 在聚 类阶段完成之后，具有最佳检测可靠性的类内节点 SU $i, \forall i \in V^{\prime}$ 采用如式(15)所示的平均共识协议进行 邻居间信息交互，交互的信息仅为每个 SU 采集到的 能量值 (即 $x_{i}(0)=e(i)$ ). 注意到每个类内节点 $\mathrm{SU} i, \forall i \in V^{\prime}$ 的邻居集已由 $N_{i}$ 变为 $N_{i}^{\prime}:=\left\{j \mid j \in V^{\prime}\right.$, $\left.\boldsymbol{A}_{i j}=1\right\}$.

最后, 在第 3 阶段, 类内的 SUs 将感知结果广播 给类外的其他 SUs.

\section{3 所提方案}

我们将上文所述的方案归纳提炼如下.

初始化: 根据式(4), 每个 SUi 以离线学习的方式 来获得分类观测量 $o(i)$.

阶段 1: 基于共识的分布式聚类

for $t=1,2 \cdots$ do

$$
\begin{aligned}
& \text { for } i=1, \cdots, N \\
& \quad \text { for } k=1, \cdots, K
\end{aligned}
$$

根据式(11)计算类归属系数 $a^{p}(i, k)$;

根据式(12)计算本地类质心 $c(i, k)$;

根据式(13)更新本地聚合拉格朗日乘子 $\lambda(i, k)$; End

$$
\text { End }
$$

End

每个 SUi 根据式(14)进行本地决策.

阶段 2: 基于共识的分布式感知

类内的每个 $\mathrm{SU} i \in V^{\prime}$ 进行本地感知，并将其初 始共识变量设为 $x_{i}(0)=e(i)$.

for $t=1,2 \cdots \mathrm{do}$

每个 $\mathrm{SU} i$ 与其一跳可达的邻居交互共识变量 $x_{i}(t)$ ，并根据式(15)定义的协议进行信息融合.

End

阶段 3: 广播

类内的 SUs 将感知结果广播给类外的其他 SUs.

\section{3 性能评估与讨论}

\section{1 仿真设置}

我们对所提的方案和已有方案的检测性能进行 对比分析与讨论. 带宽 $W$ 取为 $10 \mathrm{MHz}$ ，感知时间为 $100 \mu \mathrm{s}$. 噪声功率谱密度为 $N_{0}=-174 \mathrm{dBm} / \mathrm{Hz}$. 如图 3 (a)所示, 我们考虑一个 $10 \mathrm{~km} \times 10 \mathrm{~km}$ 的正方形区域, 1 个 PU 位于中心, 36 个 SUs 均匀分布在正方形区域 内. 主用的发射功率设为 $200 \mathrm{~mW}$. 路径损耗因子为 4, 阴影衰落标准差为 $10 \mathrm{~dB}$, 多径衰落的均值为 1 .
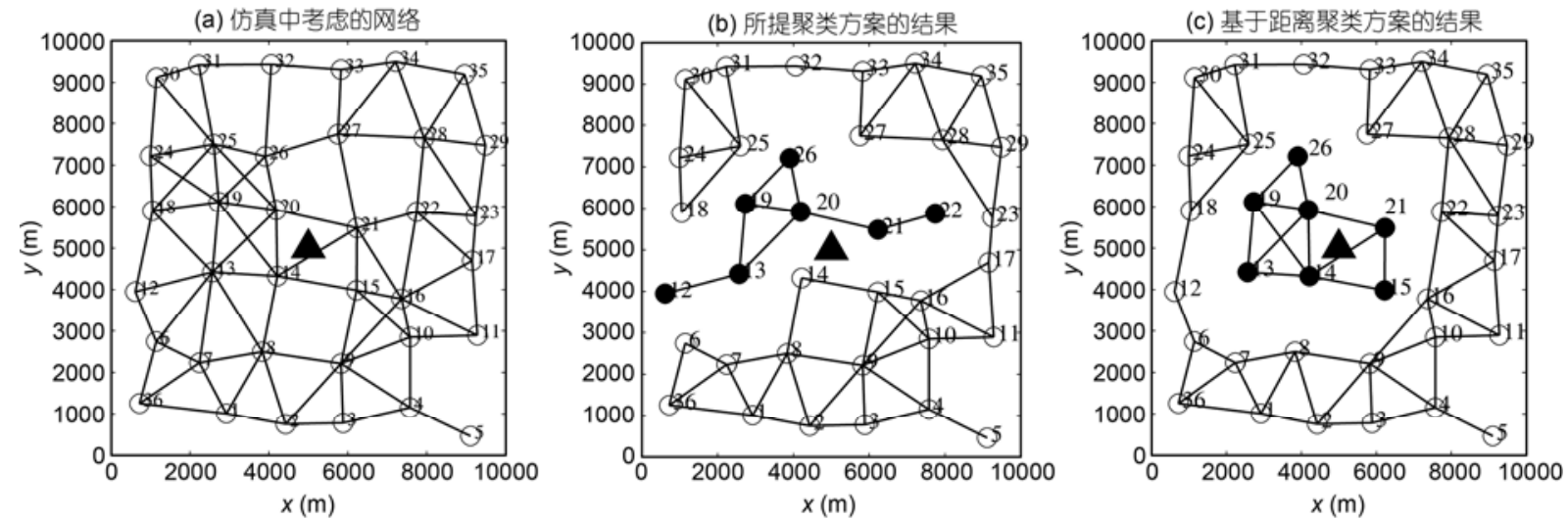

图 3 仿真所使用的网络以及不同聚类方案的结果

图中三角形表示 PU, 空心的圆圈表示 SU, 实心的圆圈表示聚类后选中的性能最佳的 SU 


\section{2 聚类结果}

图 3(b)给出了本文所提基于共识的分布式聚类 方案的结果, 图中 7 个 SUs 被选中作为潜在最佳检测 性能的用户. 作为比较, 我们在图 3(c)中给出了常见 的基于距离的分布式聚类方案的结果, 其中距离 $\mathrm{PU}$ 最近的 7 个 SUs 被选中作为潜在最佳检测性能的用 户. 图 3(b)和图 3(c)的差别源于: 基于距离的方案仅 考虑大尺度路径损耗对检测性能的影响, 而所提方 案综合考虑了路径损耗、阴影衰落和多径瑞利衰落的 影响.

\section{3 接收机工作特性曲线(ROC)对比}

在图 4 中, 我们比较了不同方案的检测性能. 图 中我们可以看到, 在给定虚警概率的情况下, 所提方 案的检测性能明显优于传统的 EGC 方案 ${ }^{[16]}$ 和基于距 离的聚类方案, 同时所提方案获得了与 OSC 方案相 近的性能, 但注意到所提方案不需要进行瞬时信噪 比的估计, 并且仅需要部分 SUs 参与感知.

\section{4 收敛性能对比}

由图 5 可以看到, 相比于所有 SUs 均参与合作的 EGC 方案和 OSC 方案, 所提部分 SUs 参与合作的方 案具有较快的收玫速度. 作为比较, 我们看到基于距 离的聚类方案与所提方案具有相近的收玫特性, 但

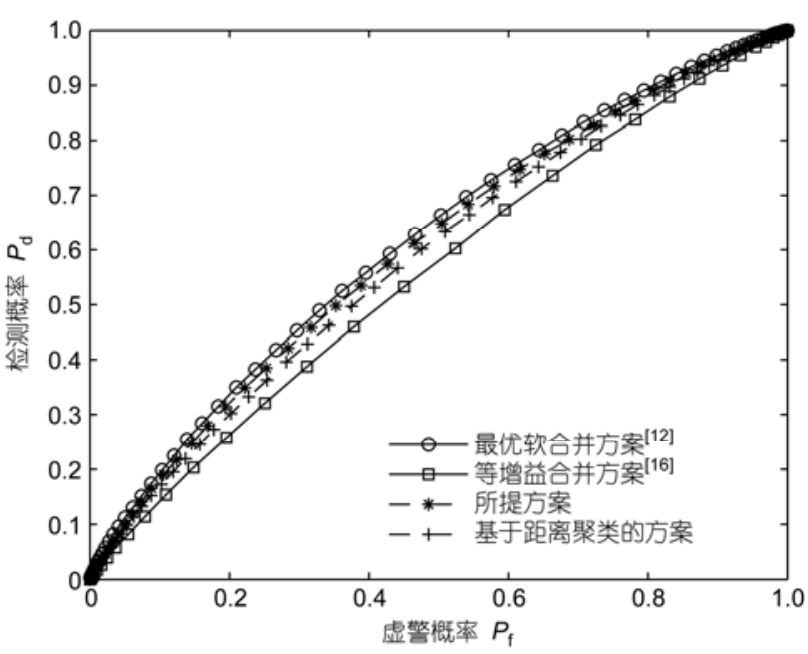

图 4 接收机工作特性曲线的比较

是所提方案不需要进行 PU 位置的估计, 从而大大降 低了算法的复杂度.

\section{5 复杂度对比}

表 1 中, 我们从参与合作的用户数、是否需要 $\mathrm{PU}$ 位置估计、是否需要接受信噪比估计等方面出发, 对 比了不同方案的复杂度. 本文所提方案的优势在于 仅需要部分用户参与合作感知、不需要 PU 位置和信 噪比的估计. 同时, 注意到相比与传统的等增益合 并 ${ }^{[16]}$ 和最优软合并 ${ }^{[12]}$ 方案, 本文所提方案存在聚类
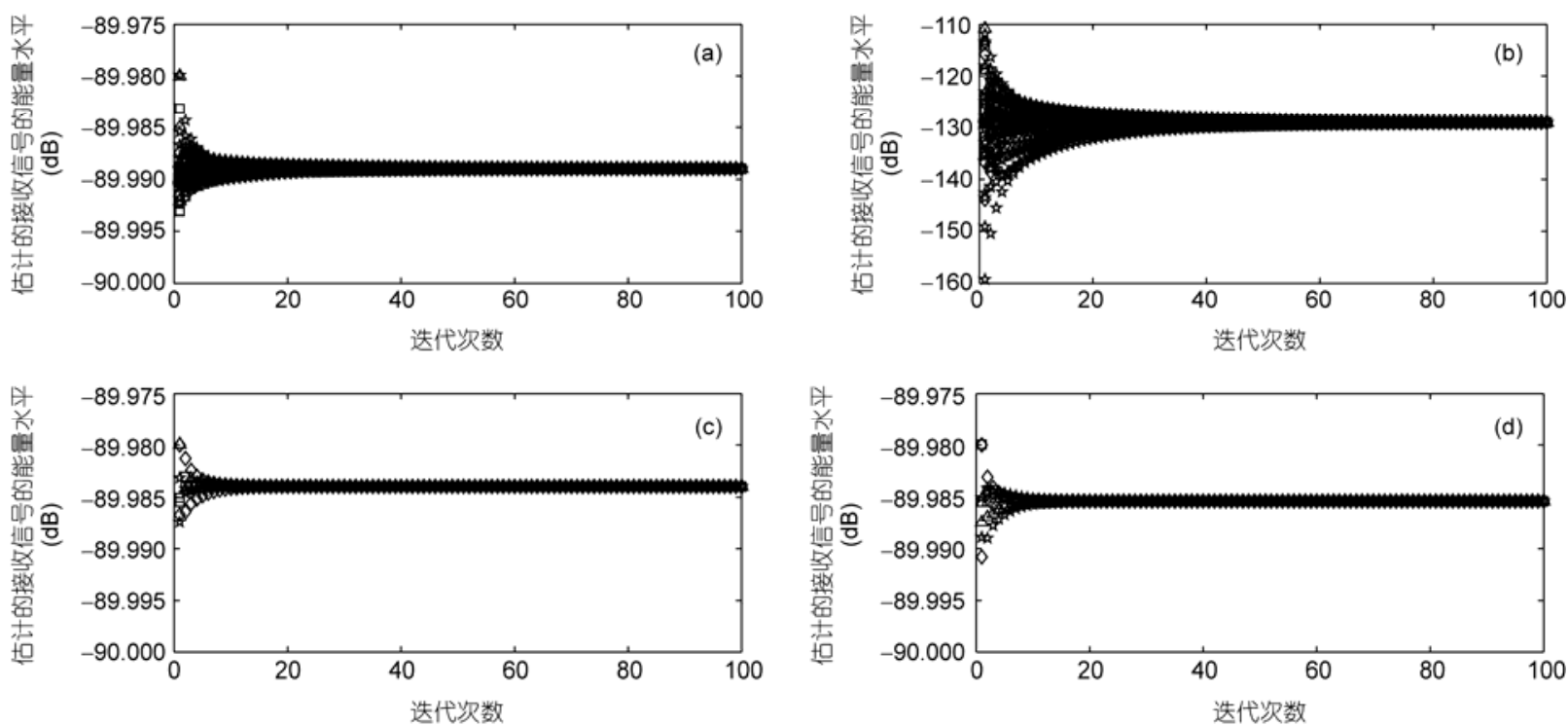

图 5 不同方案收敛性能的比较

（a）分布式等增益合并 ${ }^{[16]}$; (b) 分布式最优软合并 ${ }^{[12]}$; (c) 所提方案; (d) 基于距离的聚类 
表 1 不同方案的复杂度对比

\begin{tabular}{|c|c|c|c|c|}
\hline & 分布式等增益合并 ${ }^{[16]}$ & 分布式最优软合并 ${ }^{[12]}$ & 基于距离的聚类 & 所提方案 \\
\hline 参与合作的用户 & 所有用户 & 所有用户 & 部分用户 & 部分用户 \\
\hline 是否需要位置估计 & 否 & 否 & 是 & 否 \\
\hline 是否需要信噪比估计 & 是 & 否 & 否 & 否 \\
\hline
\end{tabular}

过程, 会带来额外的复杂度. 然而, 该聚类过程所需 的观测量可以通过离线学习的方式得到; 同时，若考 虑静态网络拓扑的, 所提方案的聚类过程仅需要在 网络初始化阶段执行.

\section{4 结论}

基于无监督学习技术与共识理论的最新进展, 本文提出了一种全分布式的合作频谱感知方案. 所
提方案主要有两个关键部分组成：分布式聚类和分 布式合作感知. 聚类环节主要实现最佳感知可靠性 节点的自组织聚集; 感知环节主要实现类内节点感 知数据的分布式融合. 数值仿真结果显示: 所提算法 在无需进行本地瞬时信噪比估计的情况下，可以获 得与最优软信息合并相近的检测性能. 潜在的研究 方向是考虑实际中感知信息的量化效应对所提算法 的影响.

\section{参考文献}

1 Tandra R, Mishra S M, Sahai A. What is a spectrum hole and what does it take to recognize one. Proc IEEE, 2009, 97: 824-848

2 Ma J, Li G, Juang B H. Signal processing in cognitive radio. Proc IEEE, 2009, 97: 805-823

3 Cabric D, Mishra S M, Brodersen R. Implementation issues in spectrum sensing for cognitive radios. In: Michael B M, ed. Proceedings of 38rd Asilomar Conference on Signals, Systems, and Computers, Pacific Grove, USA, 2004. 772-776

4 Sahai A, Tandra R, Mishra S M, et al. Fundamental design tradeoffs in cognitive radio systems. In: Milind B, Anant S, Kitti H, eds. Proceedings of the first international workshop on Technology and policy for accessing spectrum, Boston, USA, 2006. 1-6

5 Duan D L, Yang L Q, Principe J C. Cooperative diversity of spectrum sensing for cognitive radio systems. IEEE Trans Signal Process, 2010, 58: 3218-3227

6 Yucek T, Arslan H. A survey of spectrum sensing algorithms for cognitive radio applications. IEEE Commun Surveys Tuts, 2009, 11: $116-130$

7 Zeng Y H, Liang Y C, Hoang A T, et al. A review on spectrum sensing for cognitive radio: Challenges and solutions. EURASIP J Adv Signal Process. 2010: 1-15

8 Shen J Y, Jiang T, Liu S Y, et al. Maximum channel throughput via cooperative spectrum sensing in cognitive radio networks. IEEE Trans Wireless Commun, 2009, 8: 5166-5175

9 Jayakrishnan U, Venugopal V V. Cooperative sensing for primary detection in cognitive radio. IEEE J Sel Topics Signal Process, 2008, 2: $18-27$

10 Peh E C, Liang Y C, Guan Y L, et al. Optimization of cooperative sensing in cognitive radio networks: A sensing-throughput tradeoff view. IEEE Trans Veh Technol, 2009, 58: 5294-5299

11 Quan Z, Ma W, Cui S G, et al. Optimal linear fusion for distributed detection via semi-definite programming. IEEE Trans Signal Process, 2010, 58: 2431-2436

$12 \mathrm{Ma}$, Zhao G, Li Y. Soft combination and detection for cooperative spectrum sensing in cognitive radio networks. IEEE Trans Wireless Commun, 2008, 7: 4502-4507

13 Miia M, Marja M, Aarne M. Cooperative spectrum sensing using quantized soft decision combining. In: Souhir D, Joao P M, eds. Proceedings of 4th International Conference on Cognitive Radio Oriented Wireless Networks and Communications, Hannover, Germany, 2009. 1-5

14 Wang B B, Liu K J R, Clancy T C. Evolutionary cooperative spectrum sensing game: How to collaborate? IEEE Trans Commun, 2010, 58: $890-900$ 
15 Saad W, Han Z, Debbah M, et al. Coalitional games for distributed collaborative spectrum sensing in cognitive radio networks. In: Artur Z, Li Q, ed. Proceedings of 28th Conference on Computer Communications, Rio de Janeiro, Brazil, 2009. 2114-2122

16 Li Z Q, Yu F R, Huang M Y. A distributed consensus-based cooperative spectrum sensing scheme in cognitive radios. IEEE Trans Veh Technol, 2010, 59: 383-393

17 Reza O S, Fax J A, Richard M M. Consensus and cooperation in networked multi-agent systems. Proc IEEE, 2007, 95: 215-233

18 Zaheer K, Janne L, Kenta U, et al. On the selection of the best detection performance sensors for cognitive radio networks. IEEE Signal Process Lett, 2010, 17: 359-362

19 Guo C, Peng T, Xu S Y, et al. Cooperative spectrum sensing with cluster-based architecture in cognitive radio networks. In: Guillem F, ed. Proceeding of 69-th Vehicular Technology Conference: VTC2009-Spring. Bacolona, Spain, 2009. 1-5

20 Pedro A F, Alfonso C, Georgios B G. Distributed clustering using wireless sensor networks. IEEE J Sel Topics Signal Process, 2011, 5: $707-724$

21 Andrea G. Wireless Communications. London: Cambridge University Press, 2005

- 动 态・

\section{高超声速飞行器壁板颤振研究取得新进展}

壁板颤振一般是指高速气流中的飞行器表面壁板结 构发生的一种自激振动现象, 具有 3 个特点: (1) 它是一种 典型的超声速/高超音速现象; (2) 气流的作用仅发生在壁 板的一侧表面; (3) 振动的振幅一般受到结构非线性的限 制, 因此壁板颤振通常不会引发迅速的破坏, 而更多的是 造成结构的疲劳损伤. 虽然壁板㗜振不像机翼㗜振那样, 一定会导致严重的飞行事故, 但是剧烈的壁板颤振将会对 壁板结构的疲劳寿命甚至飞行器的飞行性能产生十分不利 的影响.

由北京航空航天大学航空科学与工程学院杨超教授、万 志强副教授和李国曙博士生撰写的《气动热-气动弹性双向 耦合的高超声速曲面壁板㗜振分析方法》一文展现了当前 在这一领域内的新进展. 该文发表在《中国科学：技术科 学》2012 年第 4 期上. 文中采用了新近提出的气动热-气动 弹性双向耦合分析思路, 对二维曲面壁板临界颤振时刻及 影响因素进行了研究.

对壁板颤振的研究始于 20 世纪 50 年代, 国内外研究人 员对此现象进行了大量的理论分析和试验研究. 对于气动 力, 目前应用较广的是活塞理论. 对于薄板结构, 在分析中 往往采用柯西霍夫平板假设和冯卡门非线性应变-位移关系. 另外, 高超声速条件下的壁板颤振分析还需要考虑热效应 的影响. 由于气动加热引起的温度变化相对于颤振响应是 一个缓慢的过程，因此以往在计算中往往采用非耦合的热 -结构理论. 然而, 随着高超声速飞行器的发展, 其壁板结 构相对柔度越来越大, 采用非耦合的热-结构分析理论是 否恰当就成为一个必须考虑的问题. 对此, 国外的研究人
员提出了热气动弹性双向耦合的分析方法, 该方法不仅能 够考虑气动热对气动弹性的影响, 也能够考虑弹性变形对 气动热的反馈影响. 此外, 目前已有的研究大都是针对平 面壁板, 对于曲面壁板颤振的研究相对较少, 因而使用双 向耦合分析思路的曲面壁板颤振研究就相当必要了.

文中建立了考虑气动热、气动弹性双向耦合的高超声 速二维曲面壁板颤振分析方法. 围绕 4 个方面开展了研究: (1) 考虑气动热与气动弹性的双向耦合, 既分析气动热对 结构刚度的影响, 又分析气动弹性变形对于气动压力分布 进而对气动热的影响；(2) 考虑结构温度随飞行时间的积 累效应, 以计算气动热、热传导并最终得到温度场的思路 计算结构的实际温度分布; (3) 考虑弦向和厚度方向非均 匀温度分布对气动弹性的影响; (4) 考虑曲面壁板的初始 变形, 并进而研究该变形对壁板颤振发生时刻的影响.

研究得到了以下结论: (1) 高超声速飞行器壁板, 由 于气动加热显著, 弹性效应明显, 需要充分考虑气动加热 和气动弹性之间的耦合效应.（2）基于更加贴近真实情况 的气动热、气动弹性双向耦合分析方法所得到的壁板颤振 分析结果, 较之基于单向耦合方法所得到的结果更严峻. (3) 双向耦合分析结果与单向耦合分析结果差异的产生, 与这两种方法所得到的热流和温度梯度不同有密切的关系. (4) 高超声速飞行器曲面壁板的初始变形对其颤振发生时 刻有较大的影响. 总体来说, 较大的初始变形会显著推迟 壁板发生颤振的时刻. 该研究得到了国家自然科学基金 (90716006, 10902006)的资助.

(本刊讯) 